\title{
Editorial
}

\section{Special Issue: Electron Paramagnetic Resonance}

\author{
Sylvain Bertaina ${ }^{1, *}$ and Hervé Vezin ${ }^{2, *(D)}$ \\ 1 CNRS, IM2NP (UMR 7334), Institut Matériaux Microélectronique et Nanisciences de Provence, \\ Aix-Marseille Université, 13013 Marseille, France \\ 2 CNRS, LASIR (UMR 8516), Laboratoire de Spectrochimie Infrarouge et Raman, Université de Lille, \\ 59655 Villeneuve d'Ascq, France \\ * Correspondence: sylvain.bertaina@im2np.fr (S.B.); herve.vezin@univ-lille.fr (H.V.)
}

Received: 12 September 2019; Accepted: 16 September 2019; Published: 23 September 2019

Electron paramagnetic resonance (EPR) is the tool of choice to probe and understand the dynamics of electron spin. Recent achievements in both theory and instrumentation have made EPR central in order to study new interdisciplinary topics. Such achievements are found in the collection of papers from the Special Issue, devoted to EPR, with contributions in technical improvement $[1,2]$, theory in physics [3], biology [2,4,5], and biochemistry [6]. This issue is articulated around three original articles and three specific reviews.

First of all, Daniella Goldfarb and co-workers [1] presented a modified sequence for distance measurements. Using a high field pulsed spectrometer and an arbitrary wave generator, they managed to implement this sequence of shaped pulses. The goal was to improve the signal noise ratio and so the accuracy of the distance between two Gd(III) rare earth ions was labeled on ubiquitin. They demonstrated a challenging low concentration protein labeled by Gd(III) distance measurements. Staying in high field EPR, Klaus Möbius and Anton Savitsky and co-workers [6] presented an impressive review on complementary high field EPR and NMR (nuclear magnetic resonance) in advance biomolecular spectroscopy. More than 600 papers were presented and classified, showing the entire history as well as recent improvements and state of the art in the domain. Pedro Schlottmann [3] presented the theory of EPR in ferromagnetically correlated heavy fermion, and more specifically, the role of the Kondo effect (screening of magnetism of impurity by conduction electrons) on EPR signals.

In the field of biology and medicine, EPR has attracted a lot of interest. Dariush Hinderberger and co-workers [4] presented an original study on the effect of $\mathrm{pH}$ on human serum albumin. Using both the continuous and pulse waves, they showed the improvement of using EPR, compared to other technics, to study the $\mathrm{pH}$-induced functional phase space. Fabien Peyrot and Nikola Babic [5] presented a review on EPR spectroscopy and imaging of evaluation of oxidative stress in vivo. They presented a recent achievement, state of the art, as well as the limitations in the field. Finally, Hirotada Fujii and co-workers [2] presented a review of brain redox imaging using EPR and Nitroxide probe. They showed how EPR imaging could be used to study brain disorders like Parkinson's and Alzheimer's diseases. They showed the superiority of EPR imaging compared to MRI in such studies.

We hope that this first Special Issue of Magnetochemistry, devoted top electron paramagnetic resonance, will be pleasant and useful to specialist and non-specialist readers. We thank the authors for providing such impressive and interesting papers and the referees for their time and valuable comments.

Author Contributions: S.B. and H.V. contributed to the writing this editorial.

Funding: This research received no external funding.

Conflicts of Interest: The authors declare no conflict of interest. 


\section{References}

1. Bahrenbeg, T.; Yang, Y.; Goldfarb, D.; Feintuch, A. rDEER: A Modified DEER Sequence for Distance Measurements Using Shaped Pulses. Magnetochemistry 2019, 5, 20. [CrossRef]

2. Fujii, H.G.; Emoto, M.C.; Sato-Akaba, H. Brain Redox Imaging Using In Vivo Electron Paramagnetic Resonance Imaging and Nitroxide Imaging Probes. Magnetochemistry 2019, 5, 11. [CrossRef]

3. Schlottmann, P. Theory of Electron Spin Resonance in Ferromagnetically Correlated Heavy Fermion Compounds. Magnetochemistry 2018, 4, 27. [CrossRef]

4. Reichenwallner, J.; Oehmichen, M.-T.; Schmelzer, C.E.H.; Hauenschild, T.; Kerth, A.; Hinderberger, D. Exploring the pH-Induced Functional Phase Space of Human Serum Albumin by EPR Spectroscopy. Magnetochemistry 2018, 4, 47. [CrossRef]

5. Babić, N.; Peyrot, F. Molecular Probes for Evaluation of Oxidative Stress by In Vivo EPR Spectroscopy and Imaging: State-of-the-Art and Limitations. Magnetochemistry 2019, 5, 13. [CrossRef]

6. Möbius, K.; Lubitz, W.; Cox, N.; Savitsky, A. Biomolecular EPR Meets NMR at High Magnetic Fields. Magnetochemistry 2018, 4, 50. [CrossRef]

(C) 2019 by the authors. Licensee MDPI, Basel, Switzerland. This article is an open access article distributed under the terms and conditions of the Creative Commons Attribution (CC BY) license (http://creativecommons.org/licenses/by/4.0/). 